\title{
Inhalation Induced Stresses and Flow Characteristics in Human Airways through Fluid-Structure Interaction Analysis
}

\author{
Kittisak Koombua and Ramana M. Pidaparti \\ Department of Mechanical Engineering, Virginia Commonwealth University, Richmond, VA 23284, USA \\ Correspondence should be addressed to Ramana M. Pidaparti,rmpidaparti@vcu.edu
}

Received 20 July 2007; Revised 21 April 2008; Accepted 22 May 2008

Recommended by Natalio Krasnogor

Better understanding of stresses and flow characteristics in the human airways is very important for many clinical applications such as aerosol drug therapy, inhalation toxicology, and airway remodeling process. The bifurcation geometry of airway generations 3 to 5 based on the ICRP tracheobronchial model was chosen to analyze the flow characteristics and stresses during inhalation. A computational model was developed to investigate the airway tissue flexibility effect on stresses and flow characteristics in the airways. The finite-element method with the fluid-structure interaction analysis was employed to investigate the transient responses of the flow characteristics and stresses in the airways during inhalation. The simulation results showed that tissue flexibility affected the maximum airflow velocity, airway pressure, and wall shear stress about $2 \%, 7 \%$, and $6 \%$, respectively. The simulation results also showed that the differences between the orthotropic and isotropic material models on the airway stresses were in the ranges of $25-52 \%$. The results from the present study suggest that it is very important to incorporate the orthotropic tissue properties into a computational model for studying flow characteristics and stresses in the airways.

Copyright ( $) 2008$ K. Koombua and R. M. Pidaparti. This is an open access article distributed under the Creative Commons Attribution License, which permits unrestricted use, distribution, and reproduction in any medium, provided the original work is properly cited.

\section{Introduction}

Flow characteristics in the human respiratory airways are very important for studying particle transportation in many applications ranging from aerosol drug therapy to inhalation toxicology as well as gas exchange in the airways. A better understanding of the stresses and strains and theirs influences on our airways is also important, since the airwaysare shaped by the complex mechanical environment even in the uterus. This complex mechanical environment continues to influence and alter the mature airways in healthy and diseased people [1]. Many in vivo and in vitro models have been developed to study the effect of the mechanical stresses on the airways. The results from these models showed that the high peak airway pressure caused airway distention and an increase in the level of an inflammatory mediator, cytokine $[2,3]$. In addition, high airway pressure can contribute to an increase in the thickness of the airway epithelial layer $[4,5]$. Since it is very difficult to experimentally measure the flow characteristics (airflow velocity, airway pressure, and wall shear stress (WSS)) and airway stresses in the real environment, the computational model can provide useful information about flow characteristics and stresses in the airways.

Many researchers have developed computational models to investigate airflow and particle deposition in the airways [6-12], including the effect of airway diseases, such as tumors [6-10], asthma [11], stenosis [13], and chronic obstructive pulmonary disease (COPD) $[12,14]$. The effects of carinal shape [15] and cartilage rings [16] were also studied. However, these models were developed assuming that the airways were rigid and could not be deformed or distended by fluid force from the airflow. In general, the airways are flexible and are composed of many soft tissues. They can also be distended by airflow during inhalation [17]. The literature review indicates that there is no computational model that considers the effect of airway tissue flexibility on the flow and tissue characteristics.

In the present study, a fluid-structure interaction (FSI) analysis along with the finite-element method was employed to investigate the flow characteristics and stress distributions in the airways during inhalation. Both orthotropic and 
isotropic material models were used to represent airway flexibility to study stress distributions in the airways. The results between the fluid analysis and FSI analysis are also compared.

\section{Materials and Methods}

The transient interactions between airflow and airways during inhalation were investigated solving two coupled sets of the governing equations with associated boundary conditions. The governing equations for airflow and airways are briefly described below.

\subsection{Governing Equations for Airflow}

The governing equations for transient airflow are NavierStokes equations on a moving mesh with the assumption of incompressible flow. These equations govern the principles of mass and momentum conservation and are described below using Einstein's repeated index convention [18].

Conservation of mass

$$
\frac{\rho_{g}}{\sqrt{g}} \frac{\partial}{\partial t}(\sqrt{g})+\rho_{g} \frac{\partial}{\partial x_{j}}\left(u_{j}-\frac{\partial \tilde{x}_{j}}{\partial t}\right)=0
$$

Conservation of momentum

$$
\frac{\rho_{g}}{\sqrt{g}} \frac{\partial}{\partial t}\left(\sqrt{g} u_{i}\right)+\rho_{g} \frac{\partial}{\partial x_{j}}\left[\left(u_{j}-\frac{\partial \tilde{x}_{j}}{\partial t}\right) u_{i}\right]=-\frac{\partial p}{\partial x_{i}}+\mu \frac{\partial^{2} u_{i}}{\partial x_{j}^{2}} .
$$

In these equations, $\tilde{x}_{i}$ represents the moving mesh location, $\sqrt{g}$ is the metric tensor determinate of the transformation, that is, the local computational control-volume size, $\rho_{g}$ is fluid density, $p$ is fluid pressure, $\mu$ is fluid viscosity, and $u$ is fluid velocity.

\subsection{Governing Equations for Airways}

The governing equations for movement of the airways during inhalation are the time-dependent structural equations and are described below using Einstein's repeated index convention [19].

Equation of motion

$$
\frac{\partial \sigma_{i j}}{\partial x_{j}}+F_{i}=\rho \frac{\partial^{2} u_{i}}{\partial t^{2}}
$$

Constitutive relations

$$
\sigma_{i j}=C_{i j k l} \varepsilon_{k l}
$$

In the equation above, $\sigma$ is the stress in each direction, $F$ is the body force, $\rho$ is density, and $u$ is the displacement, $C$ is the elasticity tensor, and $\varepsilon$ is the strain in each direction.

\subsection{Computational Method}

The effect of fluid pressure on a structure is significant, especially if the structure is flexible such as human airways.

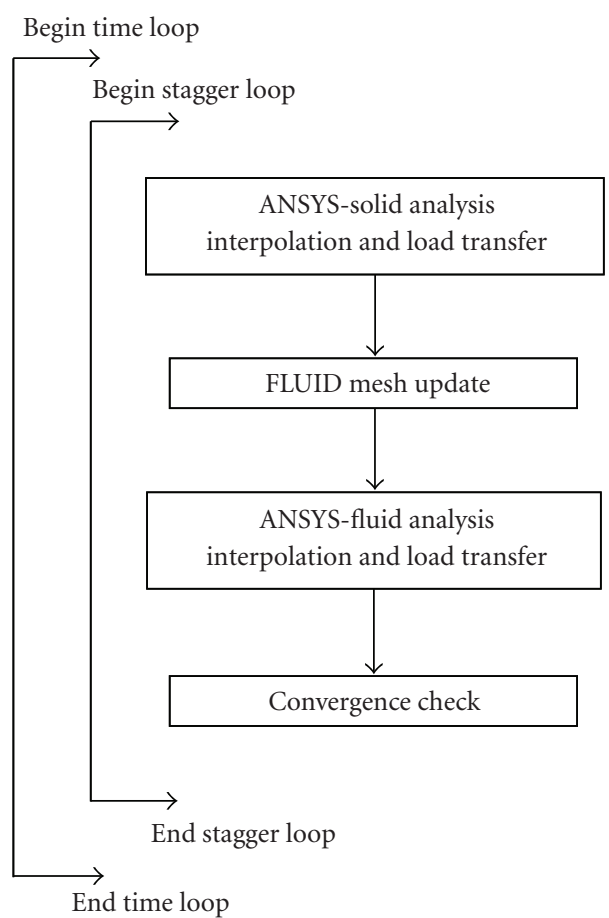

FIgURE 1: A diagram of fluid-structure-interaction algorithm.

The finite-element software ANSYS [20] was employed to solve this fluid-structure interaction problem. The solution to the fluid-structure interaction problem can be obtained by solving the governing equations for airflow and airways consecutively. At each time step, the algorithm begins by solving the airflow equations to obtain fluid pressure. Airway equations are then solved for the displacement using the fluid pressure as an external force. The airflow equations are then solved again to obtain the fluid pressure after the airway displacement changes the fluid boundaries. This loop continues until both fluid pressure and airway displacement converge for each time period (see Figure 1).

\subsection{Computational Domains}

This study focused mainly on airway generations 3 to 5 for two reasons. First, these airway generations have less cartilage plates and no rings when they are compared to the proximal generations; therefore the airways can be assumed to be smooth [21]. Second, the diameters of these airways do not change as a function of a lung volume but depend on a transmural pressure across the airways [17]. The geometric dimensions of airway generations 3 to 5 (see Figure 2) used in this study were based on the ICRP [22] tracheobronchial geometry, and airway thickness for each generation was based on measurements by Habib et al. [23]. The branching angle of the bifurcation was $70^{\circ}$ based on the morphological data of Horsfield and Cumming [24]. The corresponding geometric diameter, length, and thickness of the bifurcation are given in Table 1. The surface geometry of the model was constructed based on the physiologically realistic bifurcation (PRB) model suggested by Heistracher and Hofmann [25]. 


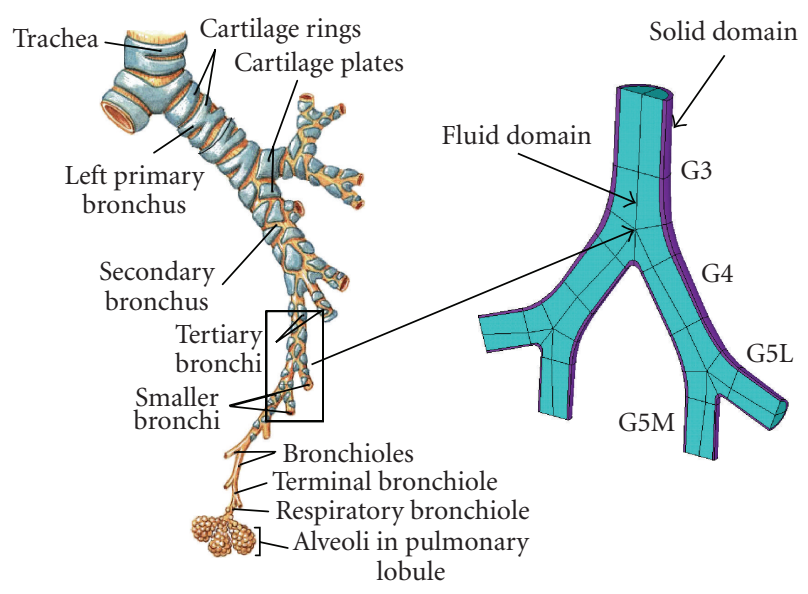

Figure 2: Geometric representations of the airway generations 3 to 5 based on ICRP [22] tracheobronchial geometry and measurements by Habib et al. [23]. These bifurcations were used as a computational domain for this study.

TABLE 1: Parameters for airway generations 3 to 5 based on ICRP [22] tracheobronchial geometry, and measurements by Habib et al. [23].

\begin{tabular}{cccc}
\hline Generation & Diameter $(\mathrm{cm})$ & Length $(\mathrm{cm})$ & Thickness $(\mathrm{cm})$ \\
\hline 3 & 0.56 & 1.10 & 0.053 \\
4 & 0.45 & 0.92 & 0.041 \\
5 & 0.36 & 0.77 & 0.024 \\
\hline
\end{tabular}

This double bifurcation geometry was previously implemented in a study by Longest and Vinchurkar [26], which evaluated the effect of transitional and turbulent flow on particle deposition in rigid airways.

\subsection{Computational Models and Boundary Conditions}

The computational domains of the bifurcation were created in ANSYS [20]. Due to symmetry, only one half of the domains was constructed. The solid domain was the airways with finite thickness, and the fluid domain was the internal volume of air in the airways. Solid elements, BRICK45 [20], were used to represent the solid domain, and fluid elements, FLUID142 [20], were used to represent the fluid domain. A structural hexahedral mesh was employed to provide a high-quality airflow solution, as suggested by Longest and Vinchurkar [27] and Vinchurkar and Longest [28]. A mesh-independence study was performed on both solid and fluid domains to confirm that a fine enough element had been used to represent the solid and fluid domains. Changes in maximum pressure and velocity were used as convergence criteria for the fluid domain, and changes in maximum displacement and von Mises stress were used as convergence criteria for the solid domain. A converged model was obtained when changes in those criteria were less than $4 \%$. Having performed the mesh-independence

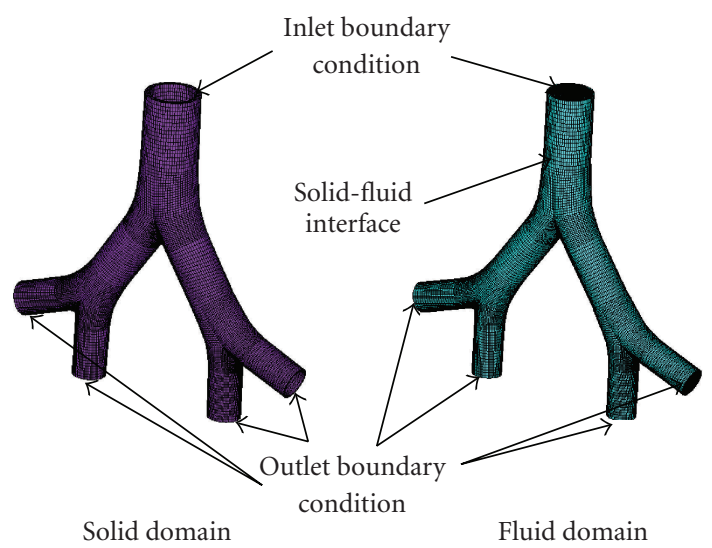

FIgURE 3: The mesh-converged finite-element model of the airway generations 3 to 5 and the boundary conditions for this study.

study, the airflow velocity from the finite-element model in the airway generation 4 was then compared with the experiment by Zhao and Lieber [29]. Good agreement was obtained between the simulation and experimental results [30]. Figure 3 shows the converged mesh that was further used for the analysis of predicting stress distributions.

The inlet boundary condition of the fluid domain was a waveform during normal inhalation [31]. The corresponding pressure was applied at the outlet of the fluid domain [32] (see Figure 4). Properties of air were assumed to be those at $27^{\circ} \mathrm{C}$. The airways were assumed to be of a homogeneous material with a density of $1365.6 \mathrm{~kg} / \mathrm{m}^{3}$ [33], a Young's modulus of elasticity in longitudinal direction of $130.89 \mathrm{kPa}$ [34], a Young's modulus of elasticity in circumferential direction of $74.07 \mathrm{kPa}$ [35], and Poisson's ratio of 0.45 [35]. A zero-displacement boundary condition was applied at the airways on both inlet and outlet of the solid domain to represent a tethering of the airways from other tissues and organs [36]. A no-slip boundary condition was defined at the fluid-structure interface (see Figure 3).

\subsection{Methods of Analysis}

Airflow velocity, airway pressure, and airway stresses were calculated in this study. Stresses in the airways during inhalation are from fluid shear force and airway pressure. Fluid shear force during inhalation creates wall shear stress (WSS) at the surface of the airways. WSS is the tangential stress at a wall due to fluid viscosity and is related to a transverse velocity gradient [37]. In contrast, airway pressure acting in the normal direction to the airways creates stresses across thickness of the airways. Stresses from the airway pressure are normal and shear stress distributions in all directions. In this study, stress distributions in the airways were analyzed employing the longitudinal and von Mises stresses. The von Mises stress is an average stress in all directions and is associated with distorting the shape of material. Any material will yield if the von Mises stress is greater than its yield strength [38]. 


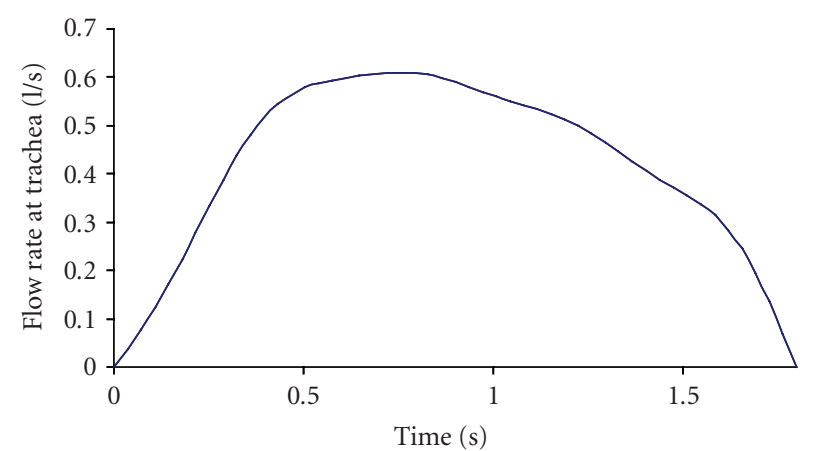

(a)

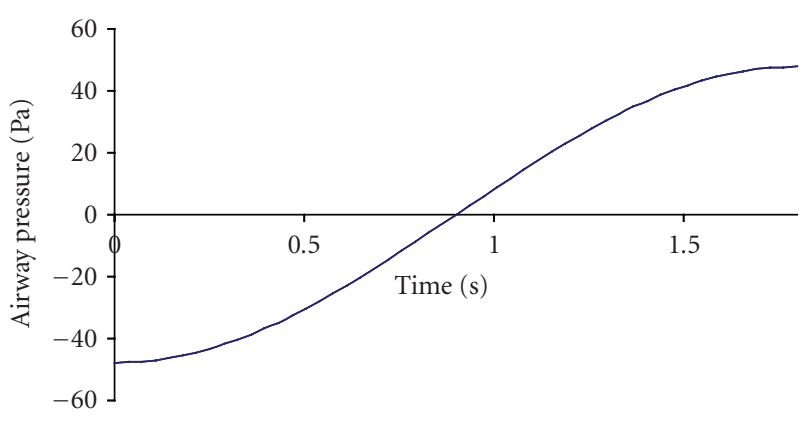

(b)

FIGURE 4: (a) Flow waveform during the normal inhalation at the trachea and (b) the corresponding airway pressure at the airway generation 5 .

Effects of airway tissue flexibility on airflow velocity, airway pressure, and WSS in the bifurcation were analyzed assuming the airways to be rigid or flexible. For the rigid model, analysis was performed only on the fluid domain. The bifurcation in this case acts like a rigid tube that cannot be deformed by fluid forces from the airflow; therefore, there is no stress in the airways. For the flexible model, analysis was performed on both fluid and solid domains. The bifurcation in this case acts like a flexible tube that can be deformed by fluid forces from the airflow. Therefore, stresses in the airways are considered in this analysis. The FSI analysis [20] was implemented for the flexible model.

To investigate the effect of tissue flexibility on stress distributions in the airways, simulations were performed using orthotropic and isotropic material models. For the orthotropic material model, the properties of the airways described in Section 2.5 were used. In contrast to the orthotropic material model, the Young's modulus of elasticity of $130.89 \mathrm{kPa}$ [34] and Poisson's ratio of 0.45 [35] was used for the isotropic material model.

\section{Results and Discussion}

The distributions of airflow velocity, airway pressure, WSS, longitudinal stress, and von Mises stress for the flexible model were evaluated only with orthotropic material model as the distributions for both flexible and rigid models are similar. The effect of tissue flexibility on airflow velocity, airway pressure, and WSS and the effect of material models on longitudinal and von Mises stresses are also discussed.

\subsection{Airflow Velocity}

The distributions of airflow velocity in the airways during the peak airflow are shown in Figure 5. The maximum airflow velocity during the peak airflow was $3.692 \mathrm{~m} / \mathrm{s}$. High airflow velocity spread throughout $\mathrm{G} 3$. Airflow velocity in the medial side of G4 was higher than that in the lateral side of G4 because the secondary flow after the bifurcation moved air toward the medial side of G4. Airflow velocity in both G3 and G4 was symmetric; however velocity profiles in G5 were

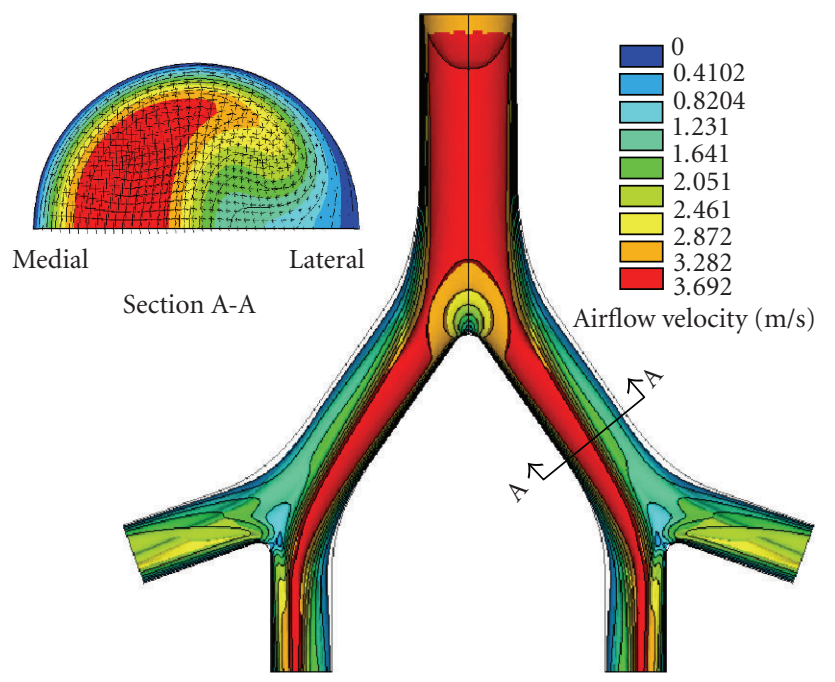

FIGURE 5: A distribution of the airflow velocity in the airway generations 3 to 5 for the orthotropic flexible model during the peak inhalation.

not symmetric. Airflow velocity in branch G5M was higher than that in branch G5L because of the high airflow velocity in the medial side of G4.

\subsection{Airway Pressure and Von Mises Stress}

The distributions of airway pressure and von Mises stress in the airways during the peak airflow are shown in Figure 6 . The maximum airway pressure during the peak airflow was $-3.209 \mathrm{~Pa}$. High airway pressure was observed at the beginning of $\mathrm{G} 3$ and the bifurcations. The pressure at the first bifurcation between G3 and G4 was about two times higher than that at the second bifurcation between G4 and G5 since the airflow that impinged the first bifurcation had more momentum (higher velocity) than the air that impinged the second bifurcation. The von Mises stress in the airways, which resulted from the airway pressure, was observed to be high at the beginning of G3 and the bifurcations. The von Mises stresses in branch G5M were higher than those in 


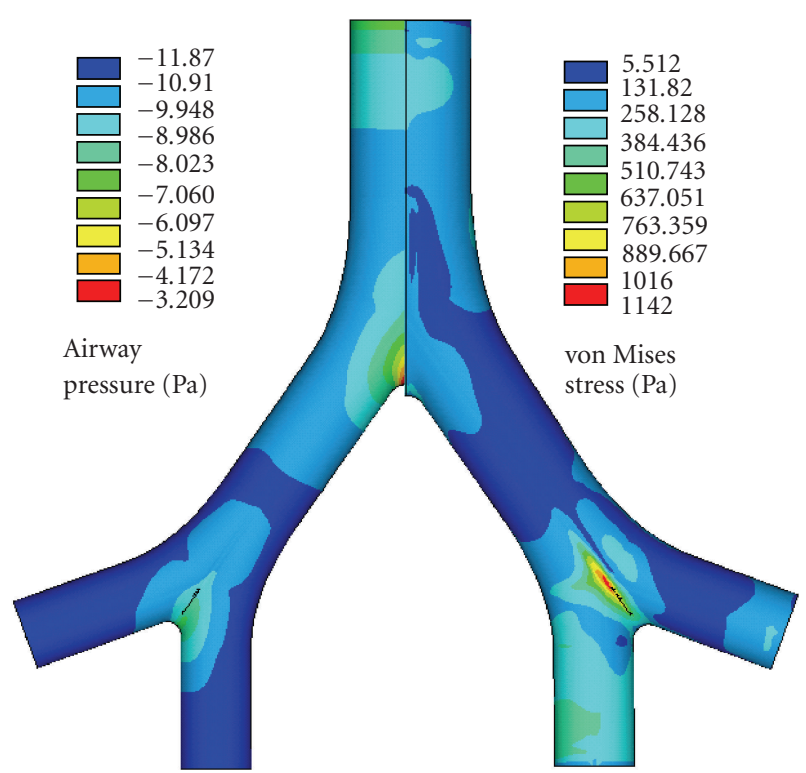

Figure 6: A distribution of the airway pressure and von Mises stress in the airway generations 3 to 5 for the orthotropic flexible model during the peak inhalation.

branch G5L. The maximum von Mises stress during the peak airflow was $1142 \mathrm{~Pa}$.

\subsection{Wall Shear Stress (WSS) and Longitudinal Stress}

The distributions of wall shear stress (WSS) at the airways and longitudinal stress in the airways during the peak airflow are shown in Figure 7. The maximum WSS, $0.4185 \mathrm{~Pa}$, occurred at the bifurcations since the velocity gradient is highest at those locations. WSS in the medial side of G4 was higher than that in the lateral side of G4, and WSS in G5M was higher than that in G5L. When the locations of the high WSS are compared with the particle deposition locations from the previous study by Longest and Vinchurkar [26], we can see that the particles deposit in the high WSS locations (see Figure 8). The longitudinal stress in the airways, which resulted from the airway pressure, was observed to be high where WSS was observed to be high. The maximum longitudinal stress during the peak airflow was $-1497 \mathrm{~Pa}$. The negative value of the longitudinal stress indicates that the length of the airways decreased during the peak airflow.

\subsection{Tissue Flexibility Effect}

Figure 9 shows the effect of tissue flexibility on the airflow velocity, airway pressure, and WSS during inhalation. As can be seen from this figure, tissue flexibility affected each flow characteristic to a different degree. Airway pressure and WSS were significantly affected by tissue flexibility. The maximum differences between the flexible and rigid models were $2 \%$, $7 \%$, and $6 \%$, for airflow velocity, airway pressure, and WSS,

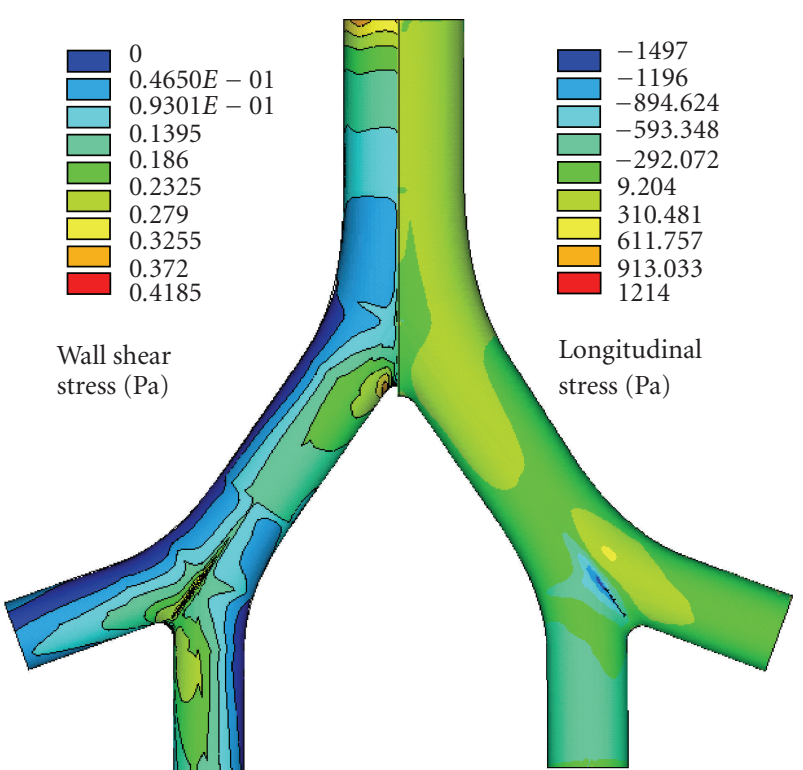

FIGURE 7: A distribution of the wall shear stress (WSS) and longitudinal stress in the airway generations 3 to 5 for the orthotropic flexible model during the peak inhalation.

respectively. The airflow velocity from the flexible model was higher than that from the rigid model for the first 0.9 seconds since the airways contracted due to the negative pressure inside the airways. However, the airflow velocity from the flexible model was lower than that from the rigid model for the last 0.9 seconds since the airways expanded due to the positive pressure inside the airways. The airway pressure from the flexible model was lower than that from the rigid model throughout the inhalation process. The movement of the airway tissue decreased the airway pressure, and the maximum differences between the flexible and rigid models were highest at the peak airflow. The increase in airway pressure associated with the rigid wall suggests that people with stiff airways, for example, elderly people [39] or asthma patients [40] can experience high airway pressure during normal inhalation. Tissue flexibility decreased WSS for the first 0.9 seconds; however, it increased WSS for the last 0.9 seconds. The effects of tissue flexibility on WSS from this study were similar to results from the previous studies in the abdominal aortic aneurysm (AAA) by Leung et al. [41] and Torii et al. [42] as well as Scotti and Finol [43]. Their results showed that tissue flexibility can increase or decrease WSS, and the influence on WSS is highly depended on AAA geometry.

\subsection{Material Model Effect}

Figure 10 shows the effect of the material models on the longitudinal and von Mises stresses. The longitudinal stress from the orthotropic material model was lower than that from the isotropic material model for entire inhalation since the isotropic material model was stiffer than the orthotropic material model. The differences between both 


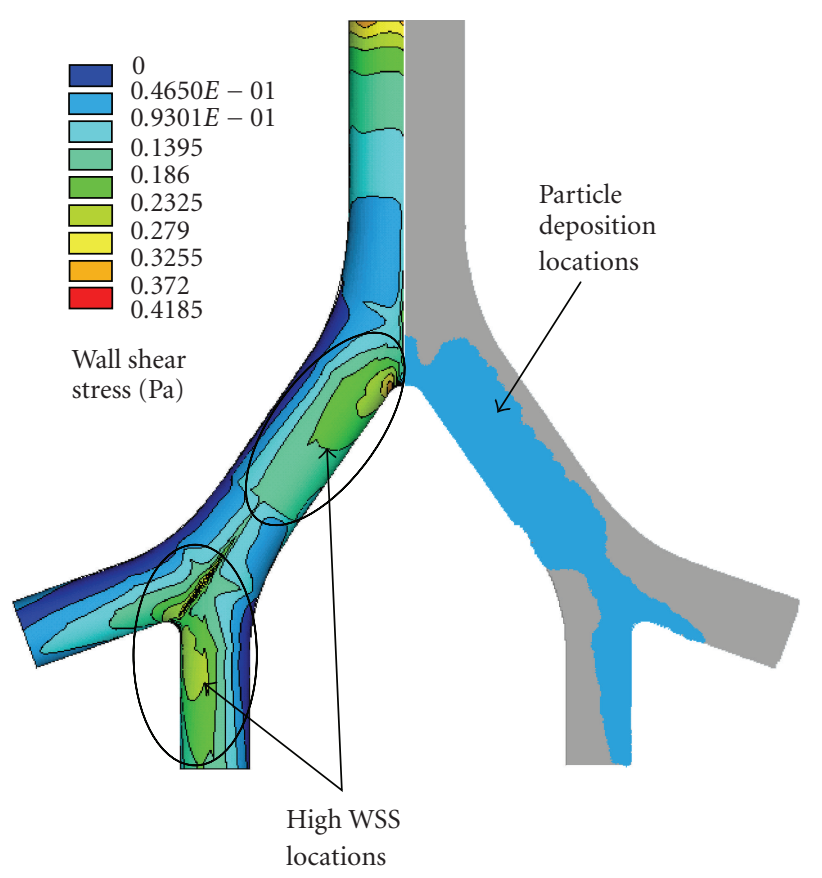

Figure 8: A distribution of wall shear stress (WSS) and locations of particle deposition in the airway generations 3 to 5 from the previous study by Longest and Vinchurkar [26].

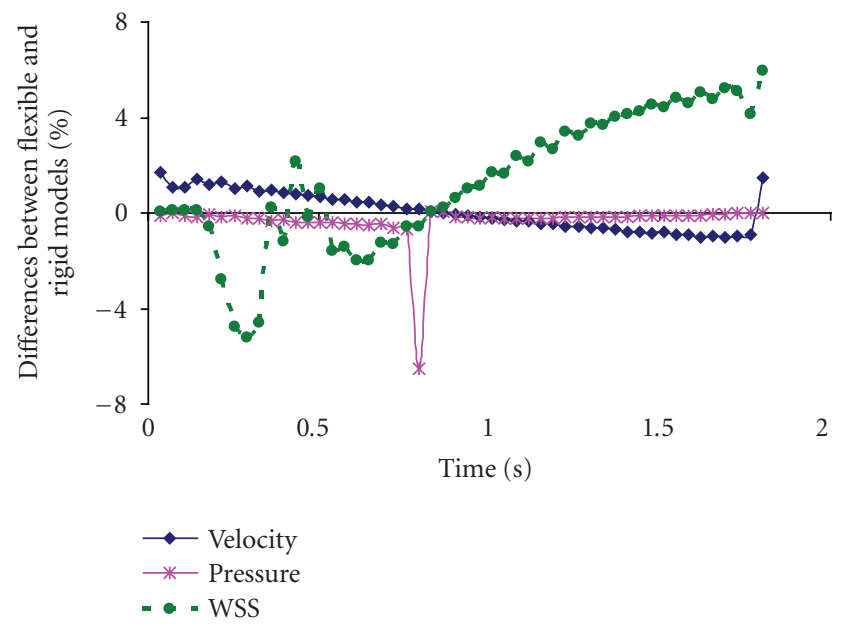

Figure 9: The tissue flexibility effect on airflow velocity, airway pressure, and wall shear stress (WSS) during the inhalation.

material models for the longitudinal stress are in the ranges of $22-52 \%$. The differences between both material models were observed to be lowest near the peak airflow. The von Mises stress from the orthotropic material model was higher than that from the isotropic material model for the first 0.9 seconds; however, the von Mises stress from the orthotropic material model was lower than that from the isotropic material model for the last 0.9 seconds. The differences between both material models for the von Mises stress are in the ranges of $25-28 \%$. The maximum difference was observed near the peak airflow.

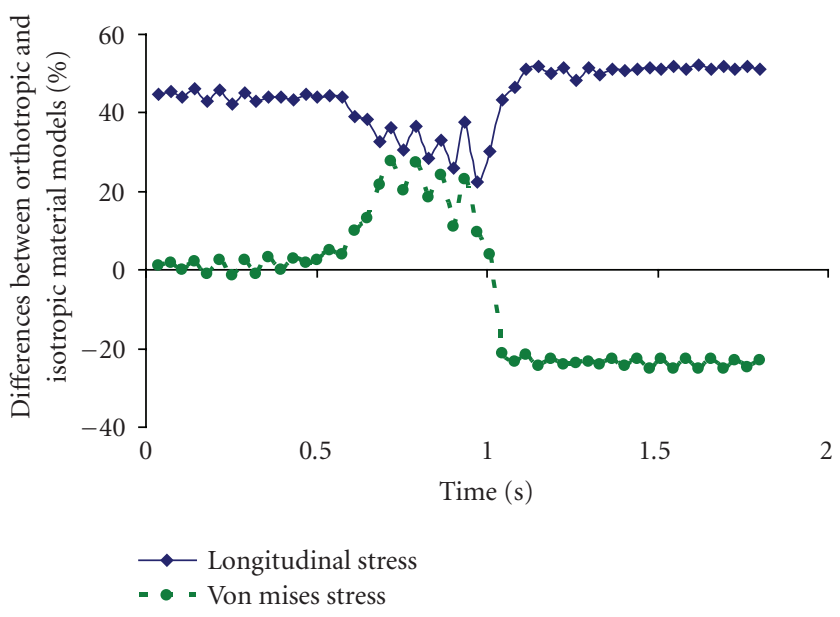

Figure 10: The material model effect on longitudinal and von Mises stresses during the inhalation.

\section{Limitations}

In this study, the airway geometry was based on an idealized ICRP [22] symmetric model. However, a study by Horsfield et al. [44] showed that airway diameters and branching airways were asymmetric. In addition, material properties of the airways in the present study were assumed to be linear. Studies by Ito et al. [45] and Smith et al. [46] showed that the airways exhibited viscoelastic properties and nonlinear dynamic behaviors. Further study is needed to investigate the effect of the airway diameters, branching angles, and airway properties on flow characteristics and stresses in the airways.

\section{Conclusions}

The airflow velocity, airway pressure, WSS, and stresses within the airway generations 3 to 5 were analyzed in this study using the finite-element method with the FSI algorithm. The analysis was performed to investigate the effects of tissue flexibility and the material model on flow characteristics and stresses in the airways during inhalation. The simulation results showed that tissue flexibility decreased the airway pressure and altered airflow velocity and WSS. The simulation results also showed that the material model of the airways significantly affected stresses in the airways. The results from this study highlight the importance of incorporating tissue flexibility along with orthotropic properties into a computational model that is developed to study flow characteristics and stresses in the airways.

\section{References}

[1] D. J. Tschumperlin and J. M. Drazen, "Chronic effects of mechanical force on airways," Annual Review of Physiology, vol. 68, pp. 563-583, 2006.

[2] S. Dhanireddy, W. A. Altemeier, G. Matute-Bello, et al., "Mechanical ventilation induces inflammation, lung injury, and extra-pulmonary organ dysfunction in experimental pneumonia," Laboratory Investigation, vol. 86, no. 8, pp. 790799, 2006. 
[3] V. M. Ranieri, P. M. Suter, C. Tortorella, et al., "Effect of mechanical ventilation on inflammatory mediators in patients with acute respiratory distress syndrome: a randomized controlled trial," The Journal of the American Medical Association, vol. 282, no. 1, pp. 54-61, 1999.

[4] M. M. Choe, P. H. S. Sporn, and M. A. Swartz, "An in vitro airway wall model of remodeling," American Journal of Physiology, vol. 285, no. 2, pp. L427-L433, 2003.

[5] M. A. Swartz, D. J. Tschumperlin, R. D. Kamm, and J. M. Drazen, "Mechanical stress is communicated between different cell types to elicit matrix remodeling," Proceedings of the National Academy of Sciences of the United States of America, vol. 98, no. 11, pp. 6180-6185, 2001.

[6] X. Guan, R. A. Segal, M. Shearer, and T. B. Martonen, "Mathematical model of airflow in the lungs of childrenII: effects of ventilatory parameters," Journal of Theoretical Medicine, vol. 3, no. 1, pp. 51-62, 2000.

[7] C. Kleinstreuer and Z. Zhang, "Targeted drug aerosol deposition analysis for a four-generation lung airway model with hemispherical tumors," Journal of Biomechanical Engineering, vol. 125, no. 2, pp. 197-206, 2003.

[8] T. B. Martonen and X. Guan, "Effects of tumors on inhaled pharmacologic drugs-I: flow patterns," Cell Biochemistry and Biophysics, vol. 35, no. 3, pp. 233-243, 2001.

[9] T. B. Martonen and X. Guan, "Effects of tumors on inhaled pharmacologic drugs-II: particle motion," Cell Biochemistry and Biophysics, vol. 35, no. 3, pp. 245-253, 2001.

[10] R. A. Segal, X. Guan, M. Shearer, and T. B. Martonen, "Mathematical model of airflow in the lungs of childrenI: effects of tumor sizes and locations," Computational and Mathematical Methods in Medicine, vol. 2, no. 3, pp. 199-213, 2000.

[11] P. W. Longest, S. Vinchurkar, and T. Martonen, "Transport and deposition of respiratory aerosols in models of childhood asthma," Journal of Aerosol Science, vol. 37, no. 10, pp. 1234$1257,2006$.

[12] X. L. Yang, Y. Liu, and H. Y. Luo, "Respiratory flow in obstructed airways," Journal of Biomechanics, vol. 39, no. 15, pp. 2743-2751, 2006.

[13] M. Brouns, S. T. Jayaraju, C. Lacor, et al., "Tracheal stenosis: a flow dynamics study," Journal of Applied Physiology, vol. 102, no. 3, pp. 1178-1184, 2007.

[14] H. Y. Luo, Y. Liu, and X. L. Yang, "Particle deposition in obstructed airways," Journal of Biomechanics, vol. 40, no. 14, pp. 3096-3104, 2007.

[15] T. B. Martonen, Y. Yang, and Z. Q. Xue, "Effects of carinal ridge shapes on lung airstreams," Aerosol Science and Technology, vol. 21, no. 2, pp. 119-136, 1994.

[16] Y. Zhang and W. H. Finlay, "Measurement of the effect of cartilaginous rings on particle deposition in a proximal lung bifurcation model," Aerosol Science and Technology, vol. 39, no. 5, pp. 394-399, 2005.

[17] A. B. Lumb, Nunn's Applied Respiratory Physiology, Butterworth-Heinemann, Oxford, UK, 2005.

[18] P. W. Longest and C. Kleinstreuer, "Computational models for simulating multicomponent aerosol evaporation in the upper respiratory airways," Aerosol Science and Technology, vol. 39, no. 2, pp. 124-138, 2005.

[19] J. N. Reddy, An Introduction to the Finite Element Method, McGraw-Hill, New York, NY, USA, 1993.

[20] ANSYS, ANSYS 10.0 User Guide, ANSYS Inc, Canonsburg, Pa, USA, 2005.

[21] M. P. Hlastala and A. J. Berger, Physiology of Respiration, Oxford University Press, New York, NY, USA, 2001.
[22] ICRP, Human Respiratory Tract Model for Radiological Protection, Elsevier, New York, NY, USA, 1994.

[23] R. H. Habib, R. B. Chalker, B. Suki, and A. C. Jackson, "Airway geometry and wall mechanical properties estimated from subglottal input impedance in humans," Journal of Applied Physiology, vol. 77, no. 1, pp. 441-451, 1994.

[24] K. Horsfield and G. Cumming, "Angles of branching and diameters of branches in the human bronchial tree," Bulletin of Mathematical Biology, vol. 29, no. 2, pp. 245-259, 1967.

[25] T. Heistracher and W. Hofmann, "Physiologically realistic models of bronchial airway bifurcations," Journal of Aerosol Science, vol. 26, no. 3, pp. 497-509, 1995.

[26] P. W. Longest and S. Vinchurkar, "Validating CFD predictions of respiratory aerosol deposition: effects of upstream transition and turbulence," Journal of Biomechanics, vol. 40, no. 2, pp. 305-316, 2007.

[27] P. W. Longest and S. Vinchurkar, "Effects of mesh style and grid convergence on particle deposition in bifurcating airway models with comparisons to experimental data," Medical Engineering \& Physics, vol. 29, no. 3, pp. 350-366, 2007.

[28] S. Vinchurkar and P. W. Longest, "Evaluation of hexahedral, prismatic and hybrid mesh styles for simulating respiratory aerosol dynamics," Computers \& Fluids, vol. 37, no. 3, pp. 317$331,2008$.

[29] Y. Zhao and B. B. Lieber, "Steady inspiratory flow in a model symmetric bifurcation," Journal of Biomechanical Engineering, vol. 116, no. 4, pp. 488-496, 1994.

[30] K. Koombua, R. M. Pidaparti, P. W. Longest, and K. R. Ward, "Computational analysis of fluid characteristics in rigid and flexible human respiratory airway models," Engineering Applications of Computational Fluid Mechanics, vol. 2, no. 2, pp. 185-194, 2008.

[31] C. L. Lafortuna, A. E. Minetti, and P. Mognoni, "Inspiratory flow pattern in humans," Journal of Applied Physiology, vol. 57, no. 4, pp. 1111-1119, 1984.

[32] D. Elad, A. Shochat, and R. J. Shiner, "Computational model of oscillatory airflow in a bronchial bifurcation," Respiration Physiology, vol. 112, no. 1, pp. 95-111, 1998.

[33] T. Sera, S. Satoh, H. Horinouchi, K. Kobayashi, and K. Tanishita, "Respiratory flow in a realistic tracheostenosis model," Journal of Biomechanical Engineering, vol. 125, no. 4, pp. 461-471, 2003.

[34] J. R. Croteau and C. D. Cook, "Volume-pressure and lengthtension measurements in human tracheal and bronchial segments," Journal of Applied Physiology, vol. 16, pp. 170-172, 1961.

[35] U. B. S. Prakash and R. E. Hyatt, "Static mechanical properties of bronchi in normal excised human lungs," Journal of Applied Physiology, vol. 45, no. 1, pp. 45-50, 1978.

[36] C. G. Plopper, S. J. Nishio, and E. S. Schelegle, "Tethering tracheobronchial airways within the lungs," American Journal of Respiratory and Critical Care Medicine, vol. 167, no. 1, pp. 2-3, 2003.

[37] E. J. Shaughnessy, I. M. Katz, and J. P. Schaffer, Introduction to Fluid Mechanics, Oxford University Press, New York, NY, USA, 2005.

[38] N. E. Dowling, Mechanical Behavior of Materials, Prentice Hall, Upper Saddle River, NJ, USA, 1998.

[39] J. K. Rains, J. L. Bert, C. R. Roberts, and P. D. Paré, “Mechanical properties of human tracheal cartilage," Journal of Applied Physiology, vol. 72, no. 1, pp. 219-225, 1992. 
[40] J. J. Fredberg, K. A. Jones, M. Nathan, et al., "Friction in airway smooth muscle: mechanism, latch, and implications in asthma," Journal of Applied Physiology, vol. 81, no. 6, pp. 27032712, 1996.

[41] J. H. Leung, A. R. Wright, N. Cheshire, et al., "Fluid structure interaction of patient specific abdominal aortic aneurisms: a comparison with solid stress models," BioMedical Engineering Online, vol. 5, article 33, pp. 1-15, 2006.

[42] R. Torii, M. Oshima, T. Kobayashi, K. Takagi, and T. E. Tezduyar, "Influence of wall elasticity in patient-specific hemodynamic simulations," Computers \& Fluids, vol. 36, no. 1, pp. 160-168, 2007.

[43] C. M. Scotti and E. A. Finol, "Compliant biomechanics of abdominal aortic aneurysms: a fluid-structure interaction study," Computers \& Structures, vol. 85, no. 11-14, pp. 1097$1113,2007$.

[44] K. Horsfield, G. Dart, D. E. Olson, G. F. Filley, and G. Cumming, "Models of the human bronchial tree," Journal of Applied Physiology, vol. 31, no. 2, pp. 207-217, 1971.

[45] S. Ito, A. Majumdar, H. Kume, et al., "Viscoelastic and dynamic nonlinear properties of airway smooth muscle tissue: roles of mechanical force and the cytoskeleton," American Journal of Physiology, vol. 290, no. 6, pp. L1227-L1237, 2006.

[46] B. A. Smith, B. Tolloczko, J. G. Martin, and P. Grütter, "Probing the viscoelastic behavior of cultured airway smooth muscle cells with atomic force microscopy: stiffening induced by contractile agonist," Biophysical Journal, vol. 88, no. 4, pp. 2994-3007, 2005. 

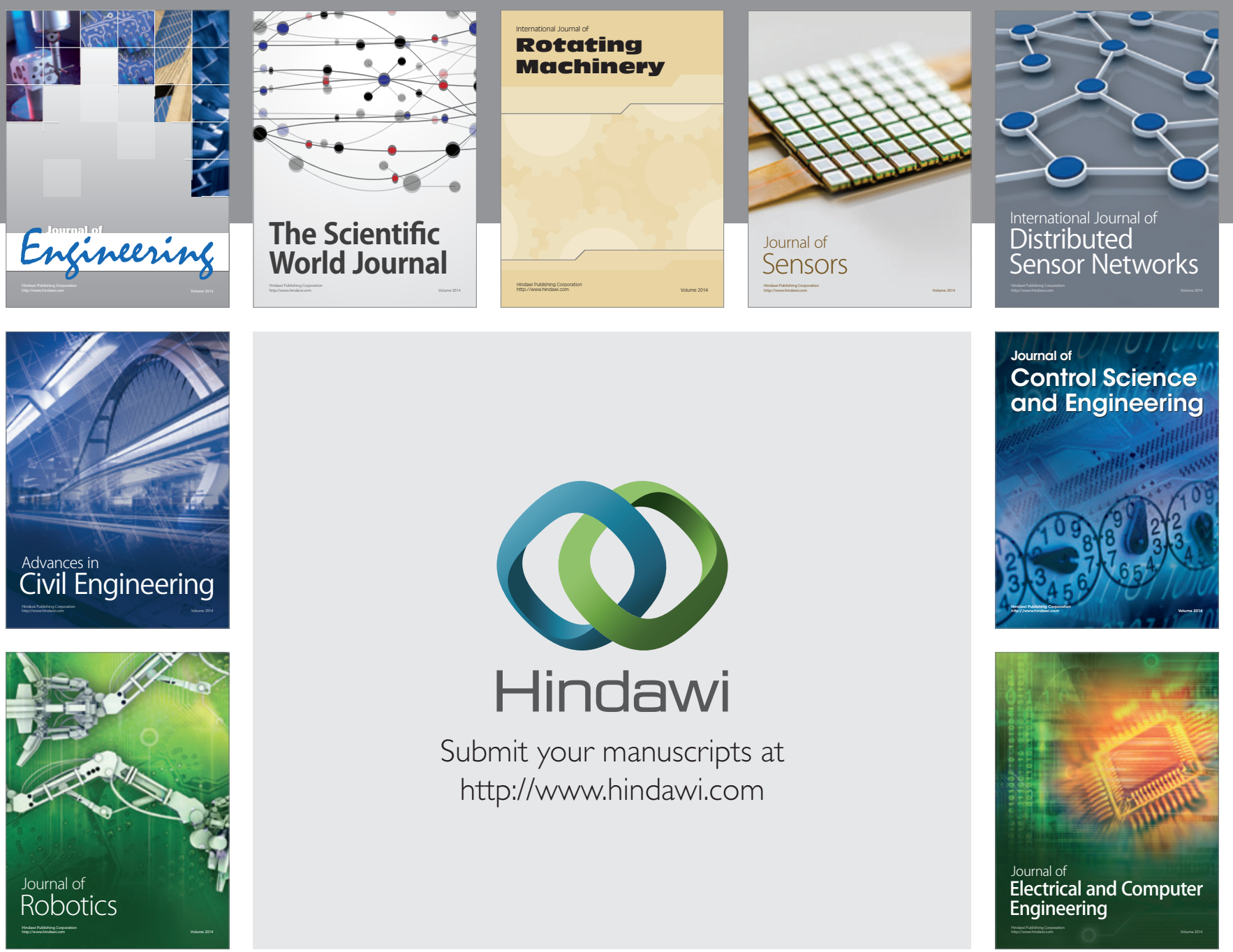

Submit your manuscripts at

http://www.hindawi.com
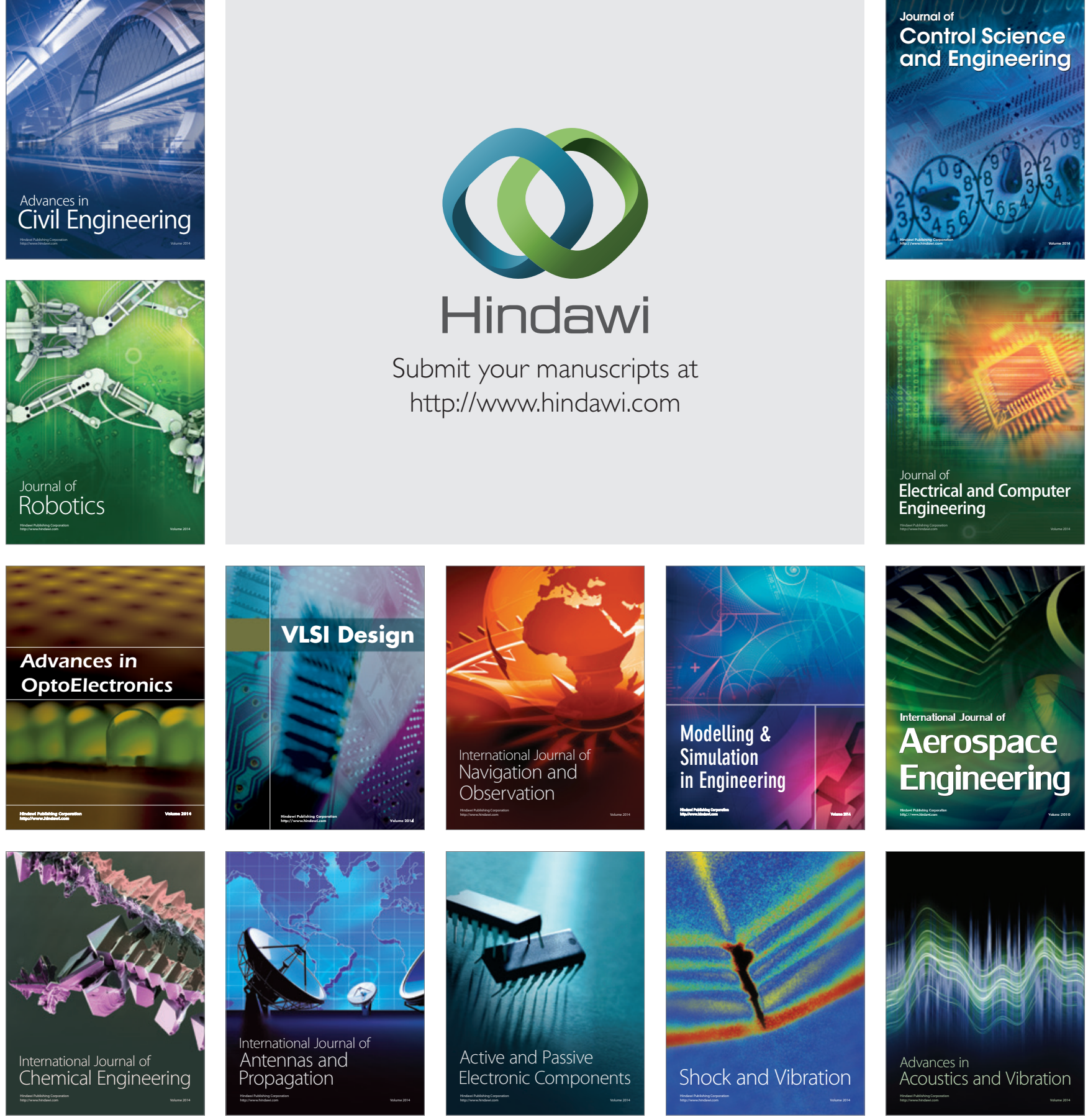